\title{
Enhancing surface integrity by high-speed extrusion machining
}

\author{
Yao Liu ${ }^{1}$ Songlin $\mathrm{Cai}^{2} \cdot$ Xinchun Shang $^{1} \cdot$ Lanhong Dai $^{3}$
}

Received: 29 December 2015 / Accepted: 25 July 2016/Published online: 8 August 2016

(C) Springer-Verlag London 2016

\begin{abstract}
High-speed machining (HSM) is an advanced machining technology to form components. However, the poor surface integrity tends to appear due to chip flow instability in HSM. It is found that the surface integrity results from the competition of shear deformation instability between in primary shear zone (PSZ) and in separating shear zone (SSZ). To improve the surface integrity of machined components, the systematic high-speed extrusion machining (HSEM) experiments of magnesium alloy AZ31B with different constraint extrusion factors (CEFs) were carried out. The instability of shear deformation in PSZ is suppressed, and the microwaves on machined surface disappear when CEF is equal to or larger than a certain value. The measurements of the machined surface show that an improvement of surface integrity is achieved if CEF exceeds a certain value. The theoretical model for HSEM was established to elucidate the critical CEF. The underlying physics of surface integrity in HSEM is further revealed. The experimental results verify the validity of the theoretical model.
\end{abstract}

Songlin Cai

caisonglin@lnm.imech.ac.cn

Lanhong Dai

lhdai@lnm.imech.ac.cn

1 School of Mathematics and Physics, University of Science and Technology Beijing, Beijing 100083, People's Republic of China

2 China Electric Power Research Institute, State Grid Corporation of China, Beijing 100192, People's Republic of China

3 State Key Laboratory of Nonlinear Mechanics, Institute of Mechanics, Chinese Academy of Sciences, Beijing 100190, People's Republic of China
Keywords High-speed extrusion machining · Surface integrity $\cdot$ Characteristic instability time $\cdot$ Chip morphology transition

\section{Introduction}

Machining operation is a versatile manufacturing process in terms of its capability to obtain designed geometrical dimensions by removing unwanted materials from a workpiece [1] High-speed machining (HSM) has become an important development area in advanced manufacturing technology due to the growing demand for enhanced manufacturing efficiency [2-4]. However, the continuous chip flow breaks down and serrated chips begin to form with the increasing cutting speed $[5,6]$. The chip morphology transition from continuous to serrated chips leads to the intense cutting force fluctuation [7-10], which is generally believed harmful for the cutting tool and surface integrity [11-14]. Therefore, the serrated chips should be suppressed in order to improve the surface integrity of machined components during HSM.

The mechanism of serrated chip has been extensively studied in the past decades. For most ductile metallic materials, the occurrence of serrated chip flow is related to the thermoplastic shear instability within primary shear zone (PSZ) [15, 16]. Considerable researches on the shear localization in PSZ have been carried out. Several classic investigations have been performed to study the onset of serrated chip flow. Recht first put forward that the serrated chips emerged in HSM if the strain hardening of materials was overtaken by the thermal softening effect within PSZ [17]. A similar approach, where the shear stress achieves a maximum in the shear stress-shear strain curve, was proposed to explain the transition from continuous to serrated chips [18]. Komanduri and Hou extended Recht's classical model to predict the onset of shear instability, 
where shear localization is imminent if the shear stress in shear band is less than or equal to the shear strength of bulk materials [19]. Semiatin and Rao first derived the quantitative critical speed for the transition from continuous to serrated chips [20]. Xie et al. showed the influence of cutting conditions on shear localization in HSM [21]. Considering the coupling system of tool-chip workpiece, Burns and Davies explained the emergence of serrated chips as a supercritical Hopf bifurcation phenomenon [22]. Aifantis et al. added the effect of strain gradient into the governing equation of PSZ and carried out the perturbation analysis of the governing equation to predict the onset of serrated chip flow [23]. Also, by using perturbation analysis, some other adiabatic shear instability critical conditions were built by considering some specific effects in HSM [24]. Recently, based on dimensional analysis and numerical simulations, Ye et al. derived an explicit expression of the critical cutting speed in terms of material properties, uncut chip thickness, and tool rake angle [25].

The fantastic pioneer works are concerned about the formation of serrated chips in HSM. The studies have demonstrated that the state of stress in PSZ has a significant effect on the chip formation in HSM. Actually, for low-speed machining (LSM), Chiffre put forward extrusion cutting to impose the extrusion stress in PSZ for controlling the chip formation process [26]. Chandrasekar and co-workers further devised a large-strain extrusion machining (LSEM) apparatus to fabricate ultrafine grain materials (UFGs) at a low cutting speed [27]. Recently, inspired by the works of Chiffre and Chandrasekar, Dai et al. developed a high-speed extrusion machining (HSEM) device to research the effect of constraint on the chip formation during HSM [28]. They stated that the chip morphology transforms from serrated to continuous chips once the constraint extrusion factor is equal to or greater than a certain value [29]. Previous work by the authors showed that constraint extrusion level affected the microstructure of chips during HSEM [30]; however, the research about the effect of extrusion on surface integrity in HSEM is vacant. In this paper, systematic HSEM experiments of magnesium alloy were carried out to explore the effect of extrusion on surface integrity. The experimental results show that an improvement of surface integrity is achieved when the constraint extrusion factor (CEF) is equal to or larger than a certain value. The theoretical model for HSEM is established to elucidate the critical CEF. The underlying physics of surface integrity in HSEM is clearly revealed by combining the experimental result with theoretical model.

\section{Experimental procedure}

The technique of HSEM has been elaborated in the authors' previous work [29]. The actual experimental setup is shown in Fig. 1, where the machining time is $4 \mathrm{~ms}$. The schematic of
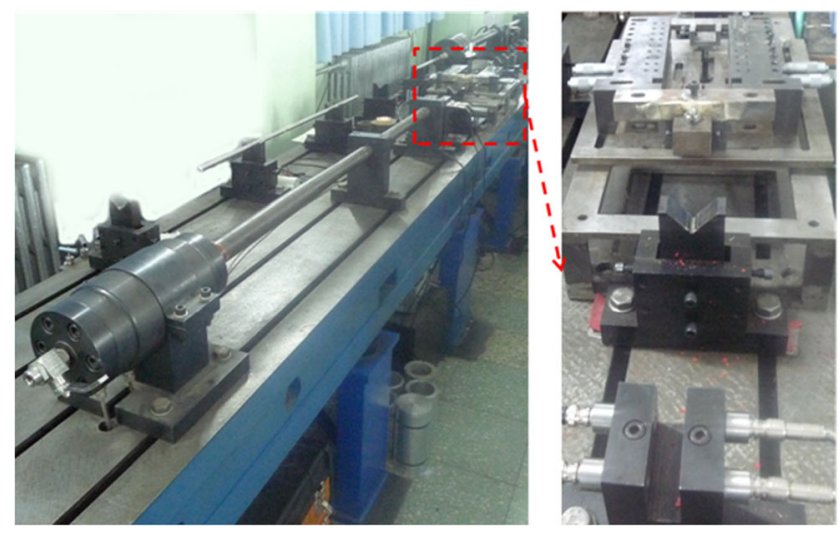

Fig. 1 The actual experimental setup

HSEM is illustrated in Fig. 2, where an orthogonal machining process is taken into consideration. The sample material used in the experiments is magnesium alloy AZ31B with chemical composition specified in Table 1. The annealing temperature of AZ31B is $345{ }^{\circ} \mathrm{C}$. The dimension of workpiece is $40 \mathrm{~mm}$ in length, $30 \mathrm{~mm}$ in width, and $2 \mathrm{~mm}$ in thickness. The microstructures of three section planes ND, RD, and TD before HSEM are shown in Fig. 2.

The process of HSEM is conducted on split Hopkinson pressure bar (SHPB), where an uncoated P10 carbide tool is used. The tool with a precut depth $t_{0}$ is cutting the workpiece at the cutting speed $V_{0}$, and the chip thickness $t_{c}$ is controlled by the constraint in HSEM. As for free machining (FM), where the constraint is useless, the chip thickness $t_{c}^{*}$ is a materialdependent parameter [28]. The wedge-shaped tool with a rake angle $\alpha$ is machining the cutting layer. Finally, the workpiece materials in the cutting layer flow out along the rake face in the form of a chip due to a process of shear in PSZ. The inclined angle $\varphi$ of PSZ is named as shear angle.

Based on the definition of CEF $\chi=\left(t_{c}^{*}-t_{c}\right) / t_{c}^{*}$ $(\chi \in[0,1])[29]$, the different constraint extrusion effects can be obtained by changing the position of constraint in the experiments. In order to explore the relationship between different CEFs and microtopographies of machined surface, the different cutting conditions for HSEM AZ31B are listed in Table 2 by adjusting the position of constraint. After cutting, chips were collected and embedded into clean resin. The lateral process was mechanically polished, and then, the polished surfaces were etched in a $5 \mathrm{~g}$ picric acid $+10 \mathrm{ml}$ water $+10 \mathrm{ml}$ acetic acid + $100 \mathrm{ml}$ ethanol solution for about $10 \mathrm{~s}$ to reveal the deformed microstructure of AZ31B. These etched specimens and machined surfaces were observed with the optical microscope (Olympus BX51M) and the scanning electron microscope (SEM FEI Sirion400NC) to examine the morphologies. The 
Fig. 2 Schematic of high-speed extrusion machining (HSEM) and microstructures of three section planes ND, RD, and TD in the sample

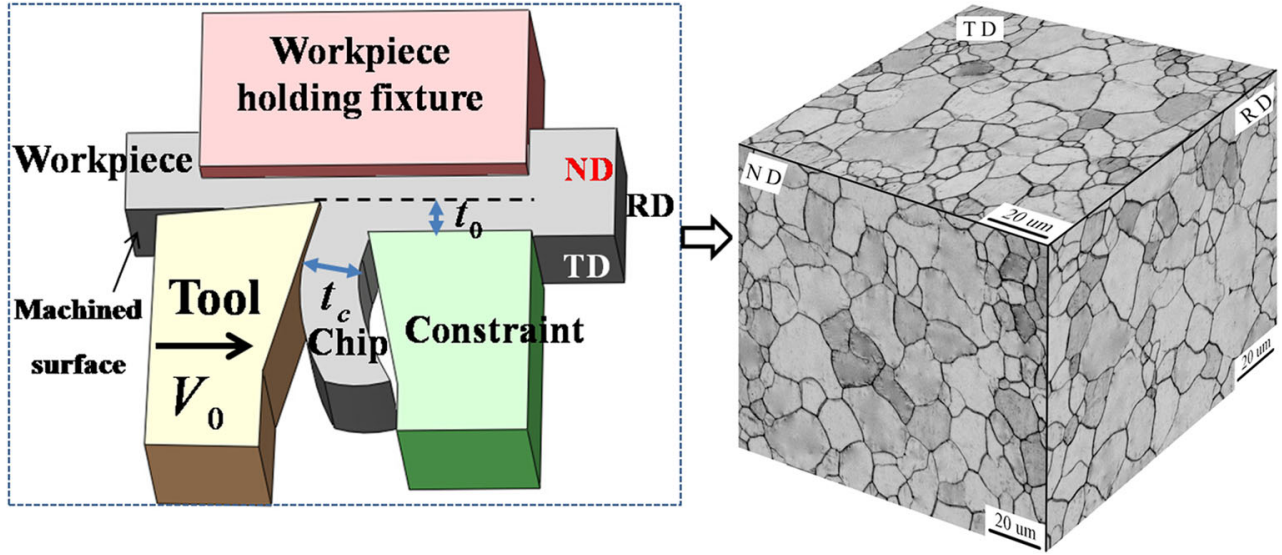

machined surfaces were further characterized by means of a ContourGT-K 3D profiler.

\section{Experimental observations}

Figure 3 shows the microstructure of chips for different CEFs in HSEM AZ31B. The transition of chip morphology from serrated to continuous is observed with the increasing CEF. As for FM (Fig. 3a), the chips are serrated due to the absence of extrusion constraint and the shear bands are obviously observed between the saw-teeth like chips. However, in the condition of HSEM, the constraint has an effect on the chip morphology and the serrations are suppressed by the constraint (Fig. 3b). When CEF reaches or exceeds a critical value, i.e., $\chi=0.56$, the thermo-plastic shear instability is totally suppressed, which leads to the disappearance of shear bands in chips (see Fig. 3c, d). In this case, homogeneous plastic deformation is realized in chips and the refined grain distributes in chips uniformly.

The occurrence of serrated chips leads to the cutting force fluctuation, decreased tool life, degradation of the surface finish, and less accuracy in machine parts during HSM. According to the experimental observations in Fig. 3, the technique of HSEM is successful to achieve the transition of chip morphology from serrated to continuous. The micro-topography of machined surface is further examined to research the effect of constraint level on the surface integrity in HSEM. As illustrated in

Table 1 Chemical composition of the magnesium alloy AZ31B

\begin{tabular}{lllllllll}
\hline Elements & $\mathrm{Mg}$ & $\mathrm{Al}$ & $\mathrm{Zn}$ & $\mathrm{Mn}$ & $\mathrm{Si}$ & $\mathrm{Cu}$ & $\mathrm{Ca}$ & Others \\
\hline Weight percent & 97 & $2.5-3.5$ & $0.6-1.4$ & 0.2 & 0.1 & 0.05 & 0.04 & $\leq 0.01$
\end{tabular}

Fig. $4 \mathrm{a}$, the periodic microwaves distribute on the machined surface uniformly for FM, as numerically predicted by Mabrouki et al. [31]. For the small CEF in HSEM, i.e., $\chi=0.32$, the periodic microwaves still emerge on the machined surface (Fig. 4b) because the serrated chips are not completely suppressed by constraint at the present constraint level (see Fig. 3b). When CEF is equal to or larger than a critical value, i.e., $\chi=0.56$, there are no periodic microwaves on the machined surface (Fig. 4c, d).

The surface integrity was further analyzed by means of a ContourGT-K 3D profiler. The digitalized scanned surfaces are shown in Fig. 5. The profiles of the machined surfaces are presented in Fig. 6 via software making a cross section of digital scanned surfaces, parallel to the cutting direction. According to the profiles of the machined surfaces in Fig. 6, the CEF induces significant variations in terms of surface integrity. The grooves are shallower with the increasing CEF, which results in a better surface integrity in HSEM. The surface roughness $R_{a}$ was measured by means of a Taylor Hobson Surtronic $25^{R}$ portable roughness tester, which is shown in Fig. 6. With the increasing CEF in HSEM, the surface roughness is smaller and a better surface integrity is achieved (see Figs. 5 and 6).

Table 2 Cutting condition in HSEM AZ31B

\begin{tabular}{lll}
\hline Cutting parameters & Notation & Value \\
\hline Rake angle & $\alpha$ & $0^{\circ}$ \\
Clearance angle & $\alpha_{2}$ & $5^{\circ}$ \\
Tool edge radius & $r_{T}$ & $10 \mu \mathrm{m}$ \\
Precut chip thickness & $t_{0}$ & $200 \mu \mathrm{m}$ \\
Cutting speed & $V_{0}$ & $10 \mathrm{~m} / \mathrm{s}$ \\
Controlled chip thickness & $t_{c}$ & $280,190,120$, and $90 \mu \mathrm{m}$ \\
Constraint extrusion factor & $\chi$ & $0,0.32,0.57$, and 0.68 \\
\hline
\end{tabular}




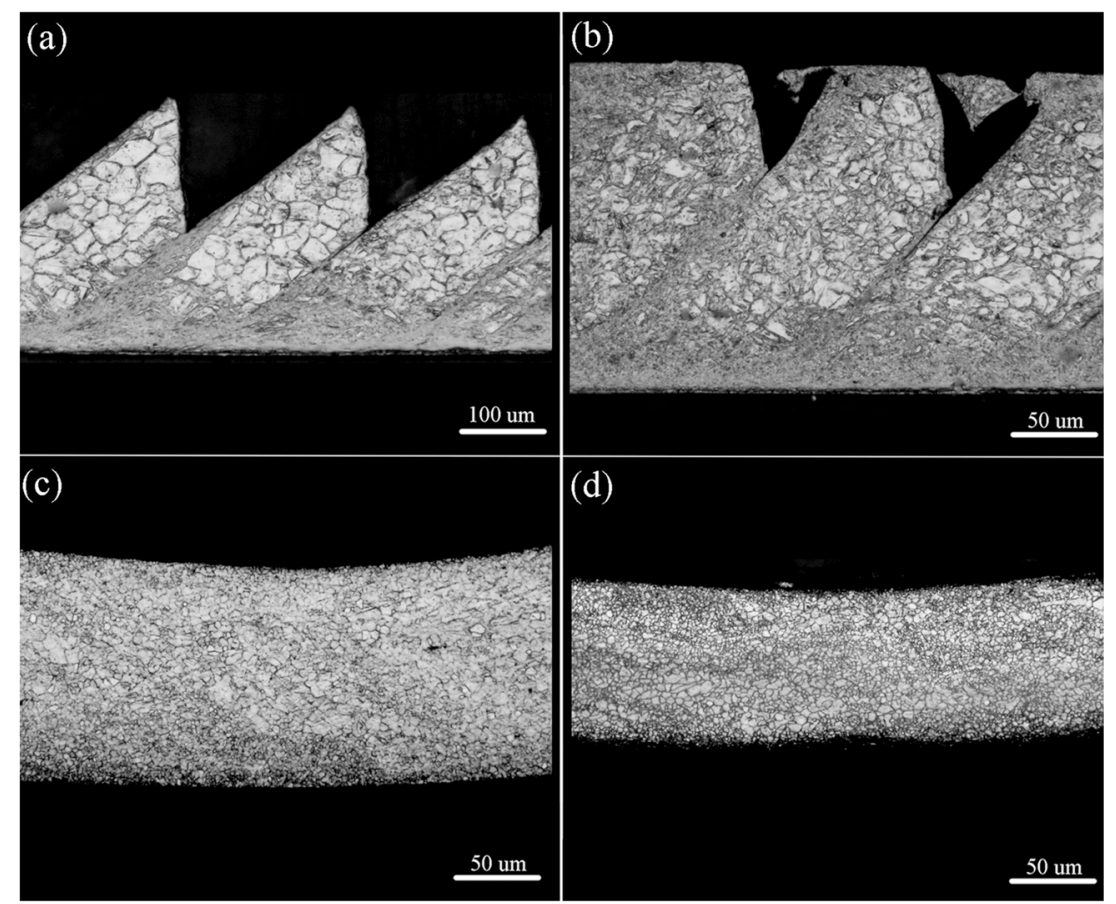

Fig. 3 The microstructures of chips for different CEFs in HSEM AZ31B: $\mathbf{a}$ CEF $=0(\mathrm{FM}), \mathbf{b} \mathrm{CEF}=0.32, \mathbf{c} \mathrm{CEF}=0.57$, and $\mathbf{d} \mathrm{CEF}=0.68$

\section{Theoretical modeling for HSEM}

\subsection{The governing equations}

As shown in the experimental results, an improvement of surface integrity can be achieved if CEF is equal to or larger than a certain value in HSEM. The theoretical model for HSEM is established here to elucidate the critical CEF. Figure 7 shows a schematic of the HSEM, where the wedge-shaped tool with a rake angle $\alpha$ is cutting the workpiece at the cutting speed $V_{0}$. The materials in the cutting layer are sheared in PSZ and then flow out along the rake face in the form of a chip. The materials in separating shear zone (SSZ) are separated from the workpiece by shear deformation within SSZ. The width of SSZ $d$ is about $t_{0} / 10$ based on experimental observations [32]. The shear deformation in PSZ and that in SSZ occur simultaneously during HSEM, which leads to the following two characteristic instability times: the characteristic instability time of $t_{p}$ in PSZ and that of $t_{s}$ in SSZ. If $t_{p}>t_{s}$, the instability of shear deformation in SSZ is before that in PSZ. The separation of materials in SSZ is ahead of the serration in PSZ. If $t_{p}<t_{s}$, the instability of shear deformation in SSZ is after that in PSZ. The materials in SSZ are not separated from the workpiece when the serration forms in PSZ, which results in a poor machined surface.

As for the characteristic instability time $t_{p}$ in PSZ, the theoretical model for $t_{p}$ put forward by Cai and
Dai [29] is used here. The spectral equation controlling the shear deformation in PSZ is the following form [29]:

$8 \alpha_{p}^{3}+8 S_{1} \alpha_{p}^{2}+2\left(S_{1}^{2}-S_{2}\right) \alpha_{p}+S_{1} S_{2}-S_{3}=0$.

In Eq. (1), $\alpha_{p}$ is the dimensionless growth rate, and the dimensionless polynomial coefficients are defined by

$\left\{\begin{array}{l}S_{1}=(1+A) k_{p}^{2}+N+C \\ S_{2}=A k_{p}^{4}+(1+N-B) k_{p}^{2}+M+C N \\ S_{3}=k_{p}^{4}+M k_{p}^{2}+M C,\end{array}\right.$

where $k_{p}$ is the dimensionless wave number and $A, B, C$, $M$, and $N$ are the dimensionless numbers which are illustrated in the work [29]. The maximum $\left(\alpha_{\mathrm{pm}}\right)$ of $\alpha_{p}$ can be obtained by solving Eqs. (1) and (2). Then, the characteristic instability time $t_{p}$ in PSZ is given by $t_{p}=1 / \alpha_{\mathrm{pm}}$.

In order to achieve the characteristic instability time $t_{s}$ in SSZ, an Eulerian coordinate system (xoy) is attached to the tool with $x$ axis parallel to the direction of cutting speed and $y$ axis normal to that direction, respectively (Fig. 7). The workpiece is considered to be thermo-viscoplastic material, and its constitutive equation is given by

$\tau=f\left(\gamma, \gamma^{\prime}, T,\right)$ 
Fig. 4 The microtopographies of machined surface for different CEFs: a CEF $=0(\mathrm{FM}), \mathbf{b}$ $\mathrm{CEF}=0.32, \mathbf{c} \mathrm{CEF}=0.57$, and $\mathbf{d}$ $\mathrm{CEF}=0.68 . \mathbf{e}-\mathbf{h}$ The local magnifications of $\mathbf{a}-\mathbf{d}$, respectively
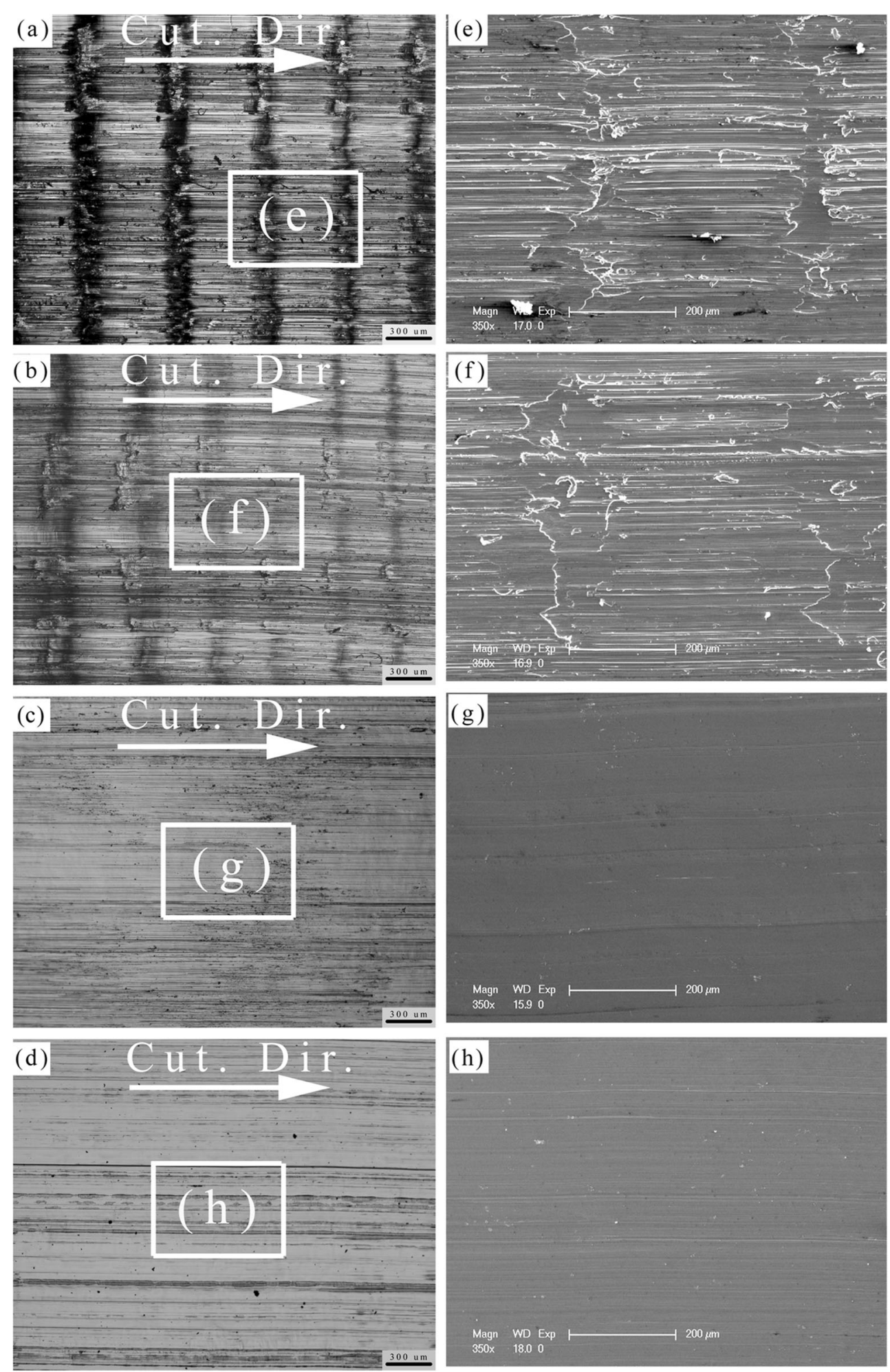

where $\tau$ is the shear stress, $\gamma$ is the shear strain, $\gamma^{\prime}$ is the shear strain rate, and $T$ is the temperature. For later use, let us introduce the following notations as did by Bai [33]: $Q=\frac{\partial f}{\partial \gamma}>0$ (strain-hardening coefficient), $R=\frac{\partial f}{\partial \gamma^{\prime}}>0$ (strain rate hardening coefficient), and $P=-\frac{\partial f}{\partial T}>0$ (thermal-softening coefficient).
In current analysis, the deformation can only occur in $x$ direction but may have a gradient in $y$ direction. Therefore, a further assumption is made for the deformation in machining; the deformation can be formulated in a one-dimensional framework, and the variables $\tau, \gamma, \gamma^{\prime}$, and $T$ depend solely on the coordinate $y$ and the time $t$. 

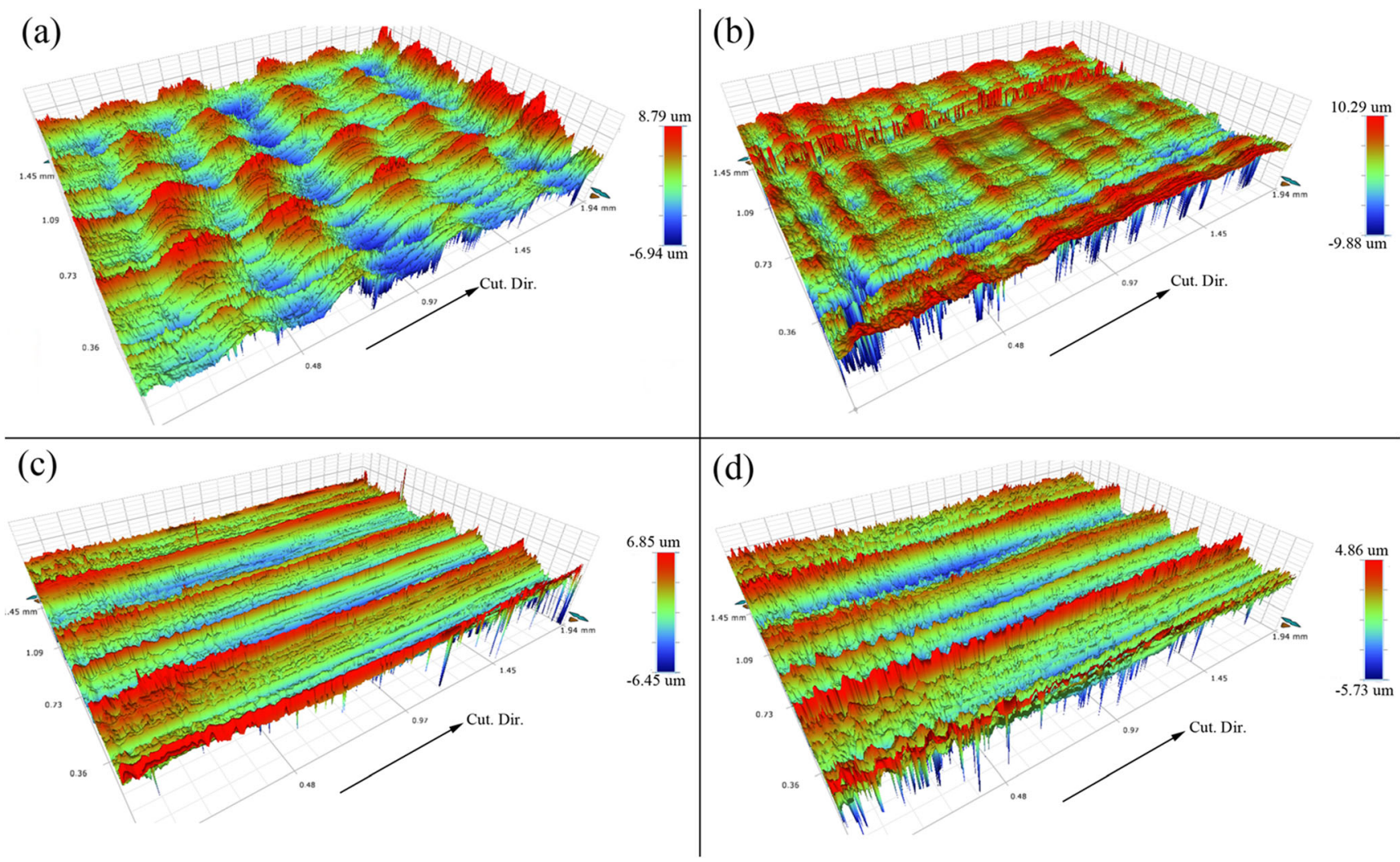

Fig. 5 The digitalized scanned surfaces for different CEFs: a CEF $=0(\mathrm{FM}), \mathbf{b} \mathrm{CEF}=0.32, \mathbf{c} \mathrm{CEF}=0.57$, and $\mathbf{d} \mathrm{CEF}=0.68$

The momentum is conserved in SSZ, so the momentum equation is given by

$\frac{\partial^{2} \tau}{\partial y^{2}}=\rho \frac{\partial \gamma^{\prime}}{\partial t}$,

where $\rho$ is the density of materials.
The conservation equation of energy in SSZ is written by

$$
\frac{\partial T}{\partial t}=\frac{\beta \tau \gamma^{\prime}}{\rho c}+\frac{k}{\rho c} \frac{T_{h}-T}{d^{2}},
$$

where $T$ is the temperature in SSZ, $T_{h}$ is the temperature around SSZ, and $d$ is the thickness of SSZ. In
Fig. 6 The profiles of the machined surfaces for different CEFs: a $\mathrm{CEF}=0(\mathrm{FM}), \mathbf{b}$ $\mathrm{CEF}=0.32, \mathbf{c} \mathrm{CEF}=0.57$, and $\mathbf{d}$ $\mathrm{CEF}=0.68$
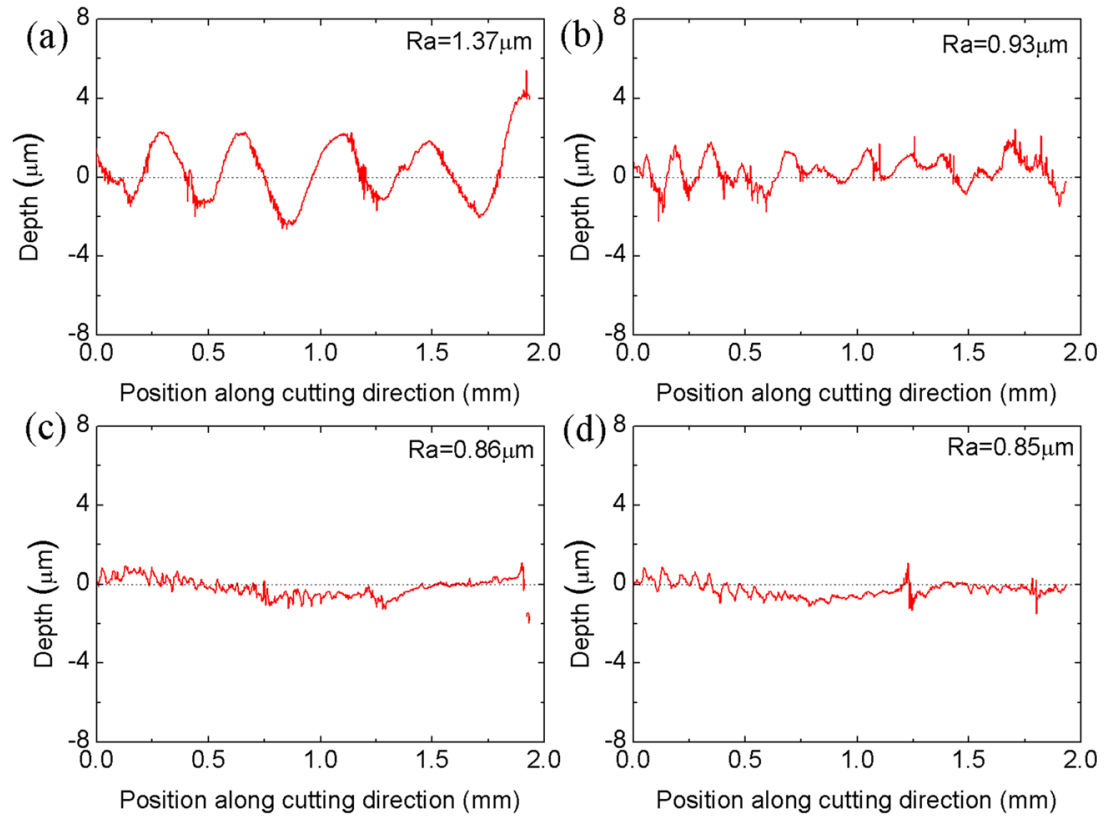


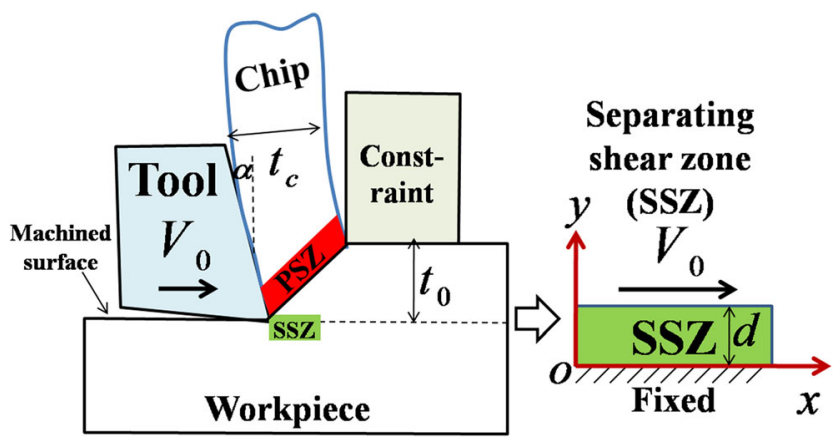

Fig. 7 The schematic of the HSEM model, where the deformation zones of PSZ and SSZ are included

Eq. (5), $\beta, k$, and $c$ are the Taylor-Quinney coefficient, thermal conductivity, and specific heat capacity, respectively. Equation (5) states that the following two physical processes control the temperature in SSZ: heat generation due to plastic working (the first term in the right side of the equation) and heat diffusion due to temperature gradient (the second term).

The chip temperature field can be estimated by finite element method (FEM) cutting simulation [34]. Recently, the temperature around SSZ $T_{h}$ is calculated from the theoretical model [35]

$T_{h}=\frac{(1-\Gamma) u_{s}}{\rho c}+T_{a}$,

$\Gamma=\frac{1}{4 Y} \operatorname{erf}(\sqrt{Y})$

$$
+(1+Y) \operatorname{erfc}(\sqrt{Y})-\frac{e^{-Y}}{\sqrt{\pi}}\left(\frac{1}{2 \sqrt{Y}}+\sqrt{Y}\right)
$$

$Y=\frac{\rho c V_{0} t_{0} \cos \alpha}{4 k(\lambda-\sin \alpha)}$,

where $\Gamma$ is the fraction of PSZ heat flowing into the workpiece, $T_{a}$ is the initial workpiece temperature, $\lambda$ is the chip thickness ratio $\left(t_{c} / t_{0}\right)$, and $u_{s}$ is the energy per unit volume dissipated within PSZ. The work has shown that $u_{s}$ increases from $2.3 \times 10^{8}$ to $4.3 \times 10^{8} \mathrm{~J} / \mathrm{m}^{3}$ when $\lambda$ is decreased from 1.4 to 0.7 [35].

The relationship between $\gamma^{\prime}$ and $\gamma$ is

$\gamma^{\prime}=\frac{\partial \gamma}{\partial t}$

The initial condition (IC) and the boundary condition (BC) governing SSZ are given by

IC $\left\{\begin{array}{l}\gamma(y, 0)=0 \\ \gamma\left(\gamma^{\prime}, 0\right)=\frac{V_{0}}{d}, \\ T(y, 0)=T_{a}\end{array}\right.$

and

$\mathrm{BC}\left\{\begin{array}{l}V(0, t)=0 \\ V(d, t)=V_{0}\end{array}\right.$.
If the specific form of constitutive relation Eq. (3) is given, we can solve simultaneously the coupled governing Eqs. (4)-(11) for the shear stress, shear strain, shear strain rate, and temperature within SSZ.

\subsection{Homogeneous deformation solution}

According to the works [36], the shear deformation before instability can be approximately regarded as a highly localized homogeneous deformation. It is necessary to seek the homogeneous solutions by assuming that shear strain rate is uniformly distributed within SSZ,

$\gamma^{\prime}=\frac{V_{0}}{d}$

The workpiece material is assumed to be rigid perfectly plastic for simplicity, and the Johnson-Cook (J-C) law was chosen to be the constitutive law of the workpiece material, which can be represented by the following formula [37]:

$\tau=\frac{1}{\sqrt{3}}\left[A+B\left(\frac{\gamma}{\sqrt{3}}\right)^{n}\right]\left[1+C \ln \left(\frac{\gamma^{\prime}}{\sqrt{3} \varepsilon_{0}}\right)\right]\left[1-\left(\frac{T-T_{a}}{T_{m}-T_{a}}\right)^{m}\right]$,

where the J-C parameters of AZ31B are listed in Table 3 [38]. Substituting the mechanical parameters of AZ31B and the cutting parameters into Eqs. (4)-(13), the partial differential Eqs. (4)-(13) are solved by using finite difference method.

Figure 8 shows the relationship between shear stress and shear strain within SSZ. The shear deformations for all CEFs are unstable. The instability of shear deformation within SSZ becomes easier with the increasing CEF. For later use, we can calculate the parameters $\tau_{0}, \gamma_{0}, \gamma_{0}^{\prime}, T_{0}, Q_{0}, R_{0}$, and $P_{0}$ through the stress-strain curve when the shear deformation begins to be unstable.

Table 3 Mechanical properties and parameters for AZ31B

\begin{tabular}{lll}
\hline Properties and parameters & Notation & Value \\
\hline Density & $\rho$ & $1770 \mathrm{~kg} \mathrm{~m}^{-3}$ \\
Elastic modulus & $E$ & $45 \mathrm{GPa}$ \\
Thermal conductivity & $k$ & $96 \mathrm{Wm}^{-1} \mathrm{~K}^{-1}$ \\
Specific heat capacity & $c$ & $1000 \mathrm{~J} \mathrm{~kg}^{-1}$ \\
& & $\mathrm{~K}^{-1}$ \\
Initial yield stress for J-C model & $A$ & $172 \mathrm{MPa}$ \\
Hardening modulus for J-C model & $B$ & $360 \mathrm{MPa}$ \\
Strain rate dependency coefficient for J-C & $C$ & 0.092 \\
$\quad$ model & & 0.456 \\
Work-hardening exponent for J-C model & $n$ & 0.95 \\
Thermal-softening exponent for J-C model & $m$ & $1 \times 10^{-3} \mathrm{~s}^{-1}$ \\
The reference strain rate for J-C model & $\varepsilon_{0}$ & $293 \mathrm{~K}$ \\
Ambient temperature for J-C model & $T_{a}$ & $890 \mathrm{~K}$ \\
Melting temperature for J-C model & $T_{m}$ \\
\hline
\end{tabular}




\subsection{Characteristic instability time within SSZ}

The physical shear localization can be related to the mathematical instability in the differential equations governing the deformation within SSZ. According to the dealing method [33], the stability analysis is simplified by seeking an inhomogeneous deformation solution with respect to small perturbations on the homogeneous solution. Such that

$$
\left[\begin{array}{c}
\tau \\
\gamma \\
\gamma^{\prime} \\
T
\end{array}\right]=\left[\begin{array}{c}
\tau_{0} \\
\gamma_{0} \\
\gamma_{0}^{\prime} \\
T_{0}
\end{array}\right]+\left[\begin{array}{c}
\tau_{*} \\
\gamma_{*} \\
\gamma_{*}^{\prime} \\
T_{*}
\end{array}\right] \exp (p t+i q y)
$$

where $\left[\begin{array}{llll}\tau & \gamma & \gamma^{\prime} & T\end{array}\right]$ are the inhomogeneous solutions of Eqs. (3)-(11), $\left[\begin{array}{llll}\tau_{0} & \gamma_{0} & \gamma_{0}^{\prime} & T_{0}\end{array}\right]$ are the homogeneous solutions, $\left[\begin{array}{llll}\tau_{*} & \gamma_{*} & \gamma_{*}^{\prime} & T_{*}\end{array}\right]$ are the small constants characterizing the magnitude of the perturbation, $q$ is the wave number, and $p$ is the growth rate. Substituting Eq. (14) into Eqs. (3)-(5) and (9), considering terms of first order in the perturbation magnitude leads to the following spectral equation:

$$
\begin{aligned}
& \rho p^{3}+\left(\frac{\beta P_{0} \gamma_{0}^{\prime}}{c}+\frac{k}{c d^{2}}+q^{2} R_{0}\right) p^{2} \\
& +\left(\frac{k R_{0}}{\rho c d^{2}}+Q_{0}-\frac{\beta P_{0} \tau_{0}}{\rho c}\right) q^{2} p+\frac{k Q_{0}}{\rho c d^{2}} q^{2} \\
& =0 .
\end{aligned}
$$

The dimensionless variables are introduced here,

$$
\left\{\alpha_{s}=\frac{k p}{c Q_{0}},{ }_{s}^{2}=\frac{k^{2} q^{2}}{\rho c^{2} Q_{0}}, A=\frac{c R_{0}}{k}, B=\frac{\beta P_{0} \tau_{0}}{\rho c Q_{0}}, C=\frac{\beta k P_{0} \gamma_{0}^{\prime}}{\rho c^{2} Q_{0}}, D=\frac{k^{2}}{\rho c^{2} d^{2} Q_{0}}\right\}
$$

and then, Eq. (15) becomes the following form:

$$
\begin{aligned}
& \alpha_{s}^{3}+\left(A k_{s}^{2}+C+D\right) \alpha_{s}^{2}+(1+A D-B) k_{s}^{2} \alpha_{s}+D k_{s}^{2} \\
& \quad=0
\end{aligned}
$$

The maximum $\left(\alpha_{\mathrm{sm}}\right)$ of $\alpha_{s}$ can be achieved by varying $k_{s}^{2}$ from 0 to $+\infty$ in Eq. (17), and then, the characteristic instability time within SSZ $t_{s}$ is obtained by $t_{s}=1 / \alpha_{\mathrm{sm}}$.

Deborah number was initially proposed by Reiner to describe the rheological behavior of materials [39]. Recently, Deborah number was also used for characterizing shear deformation instability of materials [40, 41]. Here, we define the effective Deborah number $D e$ as follows:

$D e=\frac{t_{s}}{t_{p}}$.

It is obvious that this Deborah number can characterize the competition between SSZ and PSZ. If $D e<1$, the materials

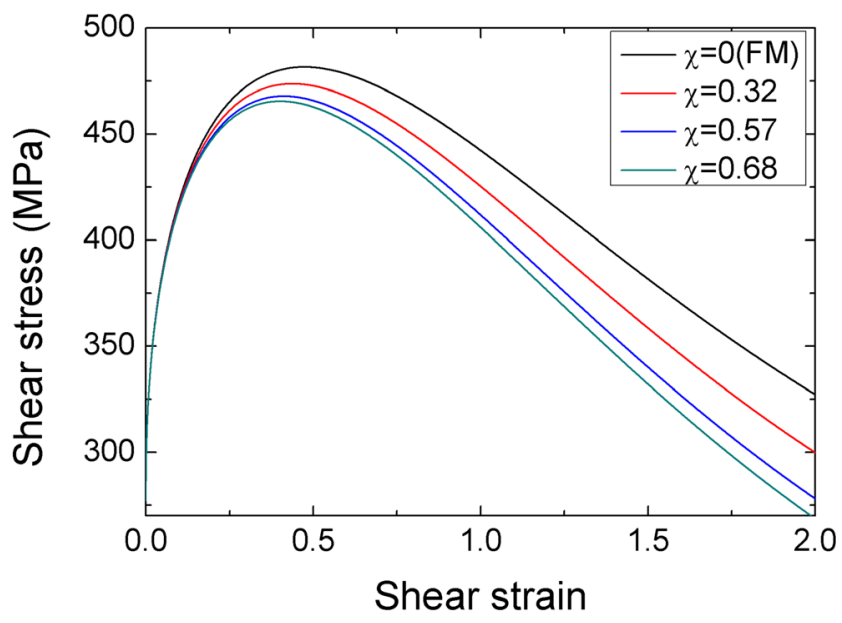

Fig. 8 The calculated stress-strain relations within SSZ for different CEFs

within SSZ tend to have fluid-like behavior; i.e., the shear deformation instability in SSZ is faster than that in PSZ. For De $>1$, the materials within SSZ are prone to have solidlike behavior; i.e., the shear deformation instability in PSZ is faster than that in SSZ.

Using the homogeneous deformation solution in Fig. 8, the variation of $D e$ with $\mathrm{CEF}$ is illustrated in Fig. 9. The poor and good surface integrity are separated by a specific constraint extrusion factor 0.5. If CEF is smaller than $0.5, D e$ is more than 1. Compared with the materials in PSZ, the materials within SSZ are more like solids. The instability of shear deformation in PSZ is before that in SSZ. The materials in SSZ are not separated completely from the workpiece during the formation of serration in PSZ, which results in a poor surface integrity. If CEF is larger than $0.5, D e$ is less than 1 . The instability of shear deformation in PSZ is after that in SSZ. The materials in SSZ are separated completely from the workpiece before the shear instability in PSZ, and an improvement of surface integrity is achieved. The deformation behaviors

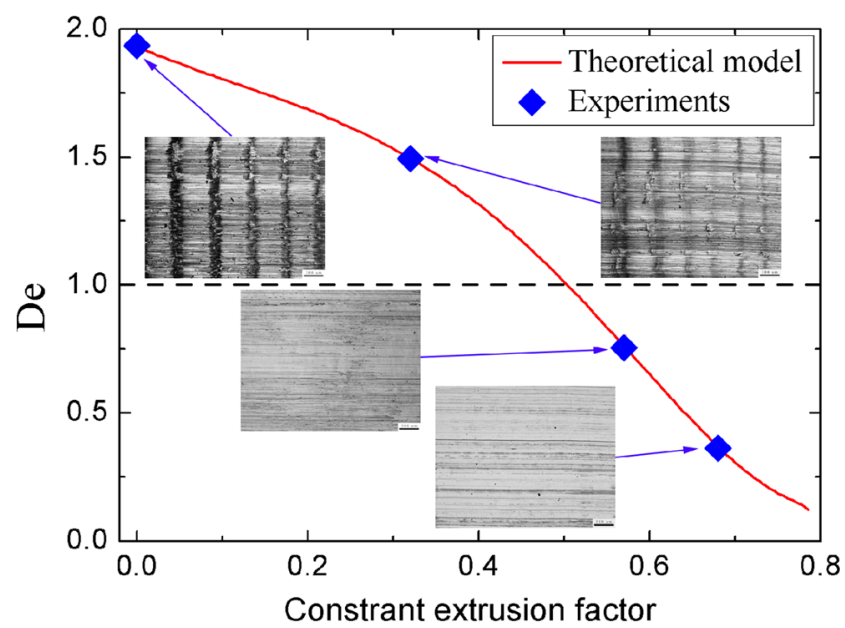

Fig. 9 Comparison of theoretical model with experimental observations for different CEFs 


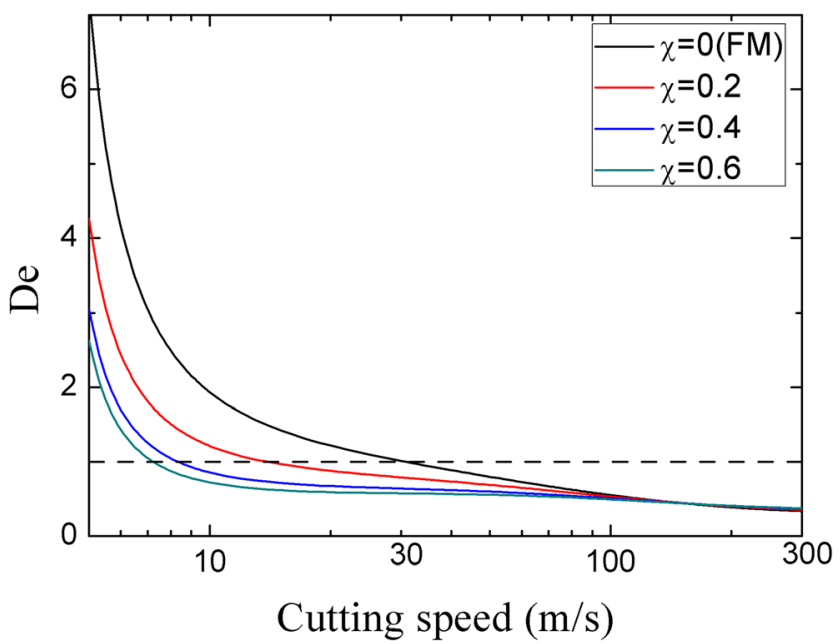

Fig. 10 The relationship between Deborah number and cutting speed for different CEFs

predicted by the theoretical model are identical to the experimental results (Fig. 9). Therefore, the present model is valid to elucidate the critical CEF, which indicates the improvement of surface integrity.

In order to further reveal the effect of constraint on surface integrity, the relationships between Deborah number and cutting speed for different CEFs are calculated by substituting the characteristic instability times into Eq. (18), which are illustrated in Fig. 10. The Deborah number decreases with the increasing cutting speed, which means that the shear deformation within SSZ is more unstable. For a given CEF, Deborah number will be less than 1 when cutting speed exceeds a certain value. That is to say, the surface integrity can be improved at higher cutting speed. The trend is in accordance with the available experimental results [42]. For a given cutting speed, Deborah number decreases with the increasing CEF. It is revealed that the constraint can promote the instability of shear deformation within SSZ.

\section{Concluding remarks}

A HSEM technique was used for improving the surface integrity. The systematic HSEM experiments of magnesium alloy AZ31B were undertaken for different CEFs. The microscopic observations of chips reveal that the shear deformation in PSZ transforms from shear band type localized deformation to homogeneous deformation with increasing CEF. The periodic microwaves on the machined surface gradually disappear when CEF exceeds a certain value. The surface roughness is smaller and a better surface integrity is achieved with the increasing CEF. Based on the experimental observations, a theoretical model for SSZ is developed to elucidate the critical $\mathrm{CEF}$, considering the competition between the characteristic instability time in PSZ and that in SSZ. The experimental results verify the rationality of the theoretical model; therefore, the theoretical model is effective to determine the critical CEF in advance.

Acknowledgments This work has been supported by the National Natural Science Foundation of China (Grant No. 11132011), Fundamental Research Funds for the Central Universities (Grant No. FRF-TP-15-101A1), China Postdoctoral Science Foundation (Grant No. 2016M591066), the Opening Fund of State Key Laboratory of Nonlinear Mechanics, the National Basic Research Program of China (Grant No. 2012CB937500), and the CAS/ SAFEA International Partnership Program for Creative Research Teams.

\section{References}

1. Subbiah S (2015) Science of machining. In: Nee AYC (ed) Handbook of manufacturing engineering and technology. Springer, London, pp. 787-810. doi:10.1007/978-1-4471-4670-4 1

2. Fang N, Pai PS, Edwards N (2013) A comparative study of highspeed machining of Ti-6Al-4V and Inconel 718 - part II: effect of dynamic tool edge wear on cutting vibrations. Int J Adv Manuf Technol 68(5-8):1417-1428

3. Fang N, Pai PS, Edwards N (2013) A comparative study of highspeed machining of Ti-6Al-4V and Inconel 718 - part I: effect of dynamic tool edge wear on cutting forces. Int J Adv Manuf Technol 68(5-8):1839-1849

4. Hamdan A, Sarhan AD, Hamdi M (2012) An optimization method of the machining parameters in high-speed machining of stainless steel using coated carbide tool for best surface finish. Int J Adv Manuf Technol 58(1-4):81-91

5. Gu L, Wang M, Chen H, Kang G (2015) Experimental study on the process of adiabatic shear fracture in isolated segment formation in high-speed machining of hardened steel. Int J Adv Manuf Technol 1-9

6. Pu CL, Zhu G, Yang S, Yue EB, Subramanian SV (2015) Effect of microstructure softening events on the chip morphology of AISI 1045 steel during high speed machining. Int J Adv Manuf Technol 1-7

7. Singh KK, Kartik V, Singh R (2015) Modeling dynamic stability in high-speed micromilling of Ti-6 $\mathrm{Al}-4 \mathrm{~V}$ via velocity and chip load dependent cutting coefficients. Int J Mach Tools Manuf 96:56-66

8. Zębala W, Kowalczyk R (2014) Estimating the effect of cutting data on surface roughness and cutting force during WC-Co turning with PCD tool using Taguchi design and ANOVA analysis. Int J Adv Manuf Technol 77(9-12):2241-2256

9. Azeem A, Feng HY (2012) Cutting force prediction for ball-end mills with non-horizontal and rotational cutting motions. Int J Adv Manuf Technol 67(5-8):1833-1845

10. Tunç L, Ozkirimli OM, Budak E (2015) Machining strategy development and parameter selection in 5-axis milling based on process simulations. Int J Adv Manuf Technol 1-18

11. Hashmi K, Zakria G, Raza M, Khalil S (2015) Optimization of process parameters for high speed machining of Ti-6Al-4V using response surface methodology. Int J Adv Manuf Technol 1-10

12. Jin D, Liu Z (2012) Effect of cutting speed on surface integrity and chip morphology in high-speed machining of PM nickel-based superalloy FGH95. Int J Adv Manuf Technol 60(9-12):893-899

13. Thakur D, Ramamoorthy B, Vijayaraghavan L (2012) Effect of cutting parameters on the degree of work hardening and tool life during high-speed machining of Inconel 718. Int J Adv Manuf Technol 59(5-8):483-489

14. Sugihara T, Takemura S, Enomoto T (2015) Study on high-speed machining of Inconel 718 focusing on tool surface topography of CBN cutting tool. Int J Adv Manuf Technol 1-9 
15. Molinari A, Soldani X, Miguélez MH (2013) Adiabatic shear banding and scaling laws in chip formation with application to cutting of Ti-6Al-4V. Journal of the Mechanics and Physics of Solids. 61(11):2331-2359

16. Cheng K, Huo D (2009) Basic concepts and theory. In: Cheng K (ed) Machining dynamics, Springer Series in Advanced Manufacturing. Springer, London, pp. 7-20. doi:10.1007/978-184628-368-0 2

17. Recht RF (1964) Catastrophic thermoplastic shear. J Appl Mech 31(2):189-193

18. von-Turkovich BF, Durham DR (1982) Machining titanium and its alloys. In: Advanced Processing Methods for Titanium, Metallurgical Society of AIME Conference, pp 257-274

19. Komanduri R, Hou ZB (2002) On thermoplastic shear instability in the machining of a titanium alloy (Ti-6Al-4V). Metall Mater Trans A-Phys Metall Mater Sci 33(9):2995-3010

20. Semiatin SL, Rao SB (1983) Shear localization during metal cutting. Mater Sci Eng 61(2):185-192

21. Xie JQ, Bayoumi AE, Zbib HM (1996) A study on shear banding in chip formation of orthogonal machining. Int J Mach Tools Manuf 36(7):835-847

22. Burns TJ, Davies MA (1997) Nonlinear dynamics model for chip segmentation in machining. Phys Rev Lett 79(3):447-450

23. Huang J, Kalaitzidou K, Sutherland JW, Aifantis EC (2007) Validation of a predictive model for adiabatic shear band formation in chips produced via orthogonal machining. J Mech Behav Mater 18(4):243-263

24. Ma W, Li X, Dai L, Ling Z (2012) Instability criterion of materials in combined stress states and its application to orthogonal cutting process. Int J Plast 30-31:18-40

25. Ye GG, Chen Y, Xue SF, Dai LH (2014) Critical cutting speed for onset of serrated chip flow in high speed machining. Int $J$ Mach Tools Manuf 86:18-33

26. De Chiffre L (1976) Extrusion cutting. Int J Mach Tool Des Res 16(2):137-144

27. Brown TL, Saldana C, Murthy TG, Mann JB, Guo Y, Allard LF, King AH, Compton WD, Trumble KP, Chandrasekar S (2009) A study of the interactive effects of strain, strain rate and temperature in LSEM of copper. Acta Mater 57(18):5491-5500

28. Cai SL, Chen Y, Ye GG, Jiang MQ, Wang HY, Dai LH (2015) Characterization of the deformation field in large-strain extrusion machining. J Mater Process Technol 216:48-58
29. Cai SL, Dai LH (2014) Suppression of repeated adiabatic shear banding by dynamic large strain extrusion machining. J Mech Phys Solids 73:84-102

30. Liu Y, Cai SL, Dai LH (2016) A new method for grain refinement in magnesium alloy: high speed extrusion machining. Mater Sci Eng A 651:878-885

31. Mabrouki T, Girardin F, Asad M, Rigal JF (2008) Numerical and experimental study of dry cutting for an aeronautic aluminium alloy (A2024-T351). Int J Mach Tools Manuf 48(11):1187-1197

32. Thakur A, Gangopadhyay S (2016) State-of-the-art in surface integrity in machining of nickel-based super alloys. Int J Mach Tools Manuf 100:25-54

33. Bai YL (1982) Thermo-plastic instability in simple shear. J Mech Phys Solids 30(4):195-207

34. Yen YC, Jain A, Altan T (2004) A finite element analysis of orthogonal machining using different tool edge geometries. J Mater Process Technol 146(1):72-81

35. Efe M, Moscoso W, Trumble KP, Dale Compton W, Chandrasekar S (2012) Mechanics of LSEM and application to deformation processing of magnesium alloys. Acta Mater 60(5):2031-2042

36. Lee WB, Zhou M (1993) A theoretical analysis of the effect of crystallographic orientation on chip formation in micromachining. Int J Mach Tools Manuf 33(3):439-447

37. Johnson GR, Cook WH (1983) A constitutive model and data for metals subjected to large strains, high strain rates and high temperatures. Proceedings of 7th International Symposium on Ballistics, Am Def Prep Ass (ADPA), Netherlands, pp 12-21

38. Feng F, Huang S, Meng Z, Hu J, Lei Y, Zhou M, Yang Z (2014) A constitutive and fracture model for AZ31B magnesium alloy in the tensile state. Mater Sci Eng A 594:334-343

39. Reiner M (1964) The Deborah number. Phys Today 17(1):62-62

40. Dai LH, Yan M, Liu LF, Bai YL (2005) Adiabatic shear banding instability in bulk metallic glasses. Appl Phys Lett 87(14)

41. Liu LF, Dai LH, Bai YL, Wei BC (2005) Initiation and propagation of shear bands in Zr-based bulk metallic glass under quasi-static and dynamic shear loadings. J Non-Cryst Solids 351(40-42):3259-3270

42. Su G, Liu Z, Li L, Wang B (2015) Influences of chip serration on micro-topography of machined surface in high-speed cutting. Int J Mach Tools Manuf 89:202-207 